\title{
Análisis del autoconcepto de adolescentes en relación al género
}

\author{
Jéssica Valeria Bayas Méndez \\ valeribayas@hotmil.com \\ Pontífice Universidad Católica del Ecuador \\ Ambato - Ecuador
}

\section{RESUMEN}

Con el objetivo de identificar el autoconcepto presente en el género femenino y en el masculino, se analizó el autoconcepto de los adolescentes en relación al género y su influencia del contexto social en esta relación, desde una investigación mixta de diseño anidado concurrente de modelo cuantitativo, de tipo descriptivo, transversal no experimental con metodología cualitativa narrativa. Participaron 112 estudiantes de 1617 años. Se les aplicó el Cuestionario del Autoconcepto AF5, que mide cinco dimensiones (académico, social, familiar, emocional y físico); y se seleccionó a diez representantes de los adolescentes que participaron en el componente narrativo - cualitativo del grupo focal respondiendo una guía de preguntas abiertas. Los resultados indicaron que existe diferencia significativa del autoconcepto en hombres y mujeres, indicando mayor autoconcepto emocional en mujeres, y en los hombres física y social; se relaciona con el resultado del grupo focal que asoció la influencia que tiene el contexto social a las diferencias del género. Estos resultados son compatibles con la literatura disponible que evidencia estas diferencias

Palabras clave: autoconcepto; género; adolescencia; contexto social. 


\title{
Analysis of the self-concept of teenagers in relation to gender
}

\begin{abstract}
The self-concept of teenagers concerning gender and its influence on the social context in this relationship was analyzed, from mixed research of concurrent nested design of a quantitative model, descriptive, non-experimental cross-sectional with qualitative narrative methodology. 112 students aged 16-17 years participated. They were given the AF5 Self-Concept Questionnaire, which measures five dimensions (academic, social, family, emotional and physical); and ten representatives of the adolescents who participated in the narrative-qualitative component of the focus group were selected by answering a guide of open questions. The results indicated that there is a significant difference in self-concept in men and women, indicating greater emotional self-concept in women, physical and social self-concept in men; it is related to the result of the focus group that associated the influence of the social context with gender differences. These results are compatible with the available literature that shows these differences.
\end{abstract}

Keywords: self-concept; gender; adolescence; social context

Artículo recibido: 30 noviembre. 2021 Aceptado para publicación: 29 diciembre 2021 Correspondencia: valeriabayas@hotmail.com Conflictos de Interés: Ninguna que declarar 


\section{INTRODUCCIÓN}

Se puede considerar al autoconcepto como la visión que tiene el sujeto de sí mismo y como es percibido por los demás, para Kinch citado por González y Taurón, 1963 el autoconcepto es la organización de cualidades, atributos y roles que el individuo se atribuye a sí mismo, emerge de la interacción social, guía e influye en la conducta futura del individuo.

Por lo que, esta conducta basada en su género también refleja cómo es percibido por sí mismo es decir los roles que debe cumplir en función a su género. La identidad de género puede proceder del aprendizaje y como fue inculcado; por ejemplo, Akers citado por Aroca, Bellver, y Alba, 2006, refiere que el aprendizaje de conductas desviadas desde los grupos primarios, secundarios y terciarios serán las que van a tener un mayor efecto sobre la conducta aquellas que ocurren antes (prioridad), duran más y ocupan más de nuestro tiempo (duración), tienen lugar con una mayor frecuencia (frecuencia) e implican a las personas con las que tenemos las relaciones más importantes y cercanas (intensidad), entendiendo que el sujeto al estar en interacción con su medio se puede decir que va adquiriendo una identidad de género que le dará paso a desarrollar conductas basadas en “como debería verse y actuar" según su género, marcando aquí las diferencias entre hombres y mujeres.

Para Loperena (2008) el autoconcepto es tomado como una construcción propia del individuo, que se va estructurando por medio de la interacción de éste con su entorno y de las experiencias positivas y negativas que internaliza en sus dimensiones: física, afectiva, intelectual y social.

Al considerar al autoconcepto como esa construcción intrínseca y extrínseca del sujeto, se podría suponer que se va modelando en sentido del género desde la infancia, ya que el infante reconoce su género y actúa con respecto a éste: (a) diferenciando los géneros; (b) asociando los comportamientos familiares y culturales que le son transmitidos; (c) reconociendo su propio género; actuando en función de ello (Gilligan 1982, citado por Rocha, 2009).

Chodorow (1984) citado por Rocha (2009) atribuye las diferencias entre los sexos al hecho de que las mujeres son educadas para servir a los demás y esto hace que las vivencias de la infancia sean diferentes y se interpreten en la vida adulta de igual forma, diferentes. El mismo autor indica que de igual manera sucede con los hombres. De esta 
manera se dará paso a desarrollar conductas basadas en "como debería verse y actuar" según su género, marcando aquí las diferencias entre hombres y mujeres.

La identidad social según Etchezahar (2014) está conformada por el autoconcepto general, que se desglosa en la valoración que un individuo realiza de los distintos roles que ocupa en un determinado contexto.

Para Vaz (2015) el autoconcepto es la manera como una persona tiende a verse de la forma como los otros la ven, se compone del feedback que el individuo guarda de su propio desempeño, también de la comparación que el individuo hace entre su comportamiento y el de aquellos que considera sus pares sociales, además se ve influenciado por el juzgamiento que un ser humano hace a su propio comportamiento teniendo en cuenta las reglas establecidas por un determinado grupo normativo al cual se encuentra vinculado; conformado o fortaleciendo su autoconcepto desde la posición del estereotipo.

Cook, citado por Solís, (2010) define que un estereotipo es una preconcepción generalizada surgida a partir de adscribir a las personas ciertos atributos, características o roles, en razón de su aparente pertenencia a un determinado grupo social, los estereotipos de género están relacionados con las características sociales y culturalmente asignadas a hombres y mujeres, a partir de las diferencias físicas basadas en su sexo, construyendo así una identidad de género.

En el proceso de la construcción de la identidad de género la sociedad suele refugiarse en los estereotipos de género. Gonzalez (1990) define que un estereotipo es una preconcepción generalizada surgida a partir de adscribir a las personas ciertos atributos, características o roles, en razón de su aparente pertenencia a un determinado grupo social. Según Melero (2010), los roles de género marcan la diferencia respecto a cómo ser, sentir y actuar tanto en mujeres y hombres condicionándolos socialmente acerca de los papeles que han desempeñado.

En las sociedades actuales existen todavía representaciones de lo femenino y lo masculino que no son coherentes con las actividades que varones y mujeres realizan en la vida cotidiana (Palladares, 2012).

Se producen, por lo tanto, actitudes discriminatorias que afectan a las personas en función de su pertenencia a un determinado estado biológico, a partir del cual se establecen diferentes conductas. Estas conductas no contribuyen a que el colectivo adolescente 
pueda "percibir con naturalidad" un estado equilibrado y equitativo de la cultura de género (Palladares, 2012).

\section{ESTRATEGIAS METODOLÓGICAS O MATERIALES Y MÉTODOS}

Esta investigación se desarrolló en base a los lineamientos metodológicos para analizar el auto concepto en relación al género de los adolescentes de bachillerato, tomando una muestra de 112 adolescentes, el estudio corresponde a una investigación aplicada, esta investigación recibe el nombre de "investigación práctica o empírica", que se caracteriza porque busca la aplicación o utilización de los conocimientos adquiridos, a la vez que se adquieren otros (Vargas, 2009).

El estudio está guiado por un paradigma post - positivista ya que el investigador puede formar parte del fenómeno de interés, el objeto de estudio influencia al investigador y viceversa, la teoría o hipótesis que sustenta la investigación influye en el desarrollo de la misma (Ramos, 2015)

La investigación se compone de un enfoque mixto, ya que estos enfoques logran obtener una mayor variedad de perspectivas del fenómeno, siendo cuantitativa para medir frecuencia, amplitud y magnitud e identificar la profundidad y complejidad como cualitativa. (Hernández, Fernández y Baptista, 2014)

Este estudio se llevó a cabo desde una metodología de investigación mixta con diseño anidado concurrente de modelo dominante al método cuantitativo, que será de alcance descriptivo - correlacional, de corte transversal no experimental, y en relación a la metodología cualitativa será narrativa.

Este estudio se llevó a cabo desde una metodología de investigación mixta con diseño anidado concurrente de modelo dominante al método cuantitativo, que fue de alcance descriptivo, de corte transversal no experimental, y en relación a la metodología cualitativa fue narrativa.

\section{Población}

La muestra estuvo conformada por 112 adolescentes ecuatorianos hombres y mujeres de 2do de Bachillerato de las Unidades Educativas Isabel de Godín y Liceo Policial de la ciudad de Riobamba de la provincia de Chimborazo comprendidos en las edades de 16 17 años de edad con una media de 16,5; y fue seleccionada siguiendo las directrices de una muestra no probabilística a conveniencia del sector y accesibilidad a los participantes. 


\section{Instrumentos}

Para la metodología cuantitativa se utilizó el cuestionario de Autoconcepto Forma 5 (AF5) elaborado por García y Musitu en el año 2001, que consta de 30 ítems formulados en términos positivos y negativos y evalúa cinco dimensiones. Académico/Laboral, que se refiere a la percepción que el sujeto tiene de la calidad del desempeño de su rol, como estudiante y trabajador, social, que se refiere a la percepción que tiene el sujeto de su desempeño en las relaciones sociales, emocional, haciendo referencia a la percepción del sujeto de su estado emocional y de sus respuestas a situaciones específicas, con cierto grado de compromiso e implicación en su vida cotidiana, familiar, que se refiere a la percepción que tiene el sujeto de su implicación, participación e integración en el medio familiar y Física haciendo referencia a la percepción que tiene el sujeto de su aspecto físico y de su condición física. Se califica mediante la escala Likert con un alfa de cronbach de 0,81 .

Para el componente cualitativo se estructuró una guía de grupo focal, donde se elaboraron 5 preguntas abiertas tomadas de las 5 dimensiones del autoconcepto y la relación que tiene cada dimensión con la sociedad y 2 preguntas explorando la sociedad actual en relación al género.

\section{Procedimiento}

Se realizó un pilotaje previo con la aplicación de los instrumentos, cuyos resultaron evidenciaron una buena aceptación de parte de los adolescentes con respecto a la validación linguiística y modo de aplicación.

La aplicación del cuestionario se realizó de forma virtual cumpliendo con los principios de bioética que incluyeron forma de consentimiento y asentimientos informados. En cuestión a la guía de grupo focal, se desarrolló de manera presencial documentando en audio y video la sesión. El análisis de información se realizó siguiendo el método científico, la parte cuantitativa se desarrolló por medio del software SPSS v.5 y el análisis cuantitativo fue por medio del software $N$ VIVO.

\section{RESULTADOS Y DISCUSIÓN}

De la muestra aplicada el 50\% son hombres y 50\% mujeres, referente a la unidad educativa el $35.7 \%$ pertenece a la Unidad Educativa Liceo Policial y el 64,3\% a la Unidad Educativa Isabel de Godín, sobre el sector de residencia el $80.4 \%$ pertenecen sector 
urbano y el $19.9 \%$ sector rural, sobre la edad 79 personas tienen 16 años y 33 sujetos tienen 17 años.

Tabla 1.

Medias de las dimensiones por género

\begin{tabular}{lccccc} 
DIMENSIONES & SEXO & N & MEDIA & DE & SIG \\
\hline \multirow{2}{*}{ Académico } & Masculino & 56 & 6.5861 & .91601 & \multirow{2}{*}{ S2 } \\
& Femenino & 56 & 6.2018 & 2.15179 & \\
\cline { 2 - 6 } Social & Masculino & 56 & 5.5618 & 1.66964 & \multirow{2}{*}{.01} \\
Emocional & Femenino & 56 & 6.7186 & 1.82290 & \\
\cline { 2 - 6 } Familiar & Masculino & 56 & 5.3780 & 1.08558 & \multirow{2}{*}{01} \\
& Femenino & 56 & 5.6277 & 1.68294 & \\
\cline { 2 - 6 } Físico & Masculino & 56 & 7.7830 & 1.30732 & \multirow{2}{*}{.27} \\
& Femenino & 56 & 7.1491 & 2.05095 & \\
\cline { 2 - 6 } & Masculino & 56 & 5.1982 & 1.68949 & \multirow{2}{*}{01} \\
\hline
\end{tabular}

En la Tabla 1. se identificó diferencias de medias en relación al género, donde la dimensión social en hombres la X es de 5.56 y en mujeres es de X 6.71 con una significancia $(\mathrm{p}<0.1)$ teniendo las mujeres mayor nivel de autoconcepto en la dimensión social que los hombres. De igual manera, la dimensión emocional en hombres es de X 5.37 y en mujeres X 5.62 ( $\mathrm{p}<0.1)$ siendo más presente el autoconcepto emocional en mujeres que en hombres. En cambio, en la dimensión físico los hombres tienen la media X 5.19 y las mujeres la media X 4.39 teniendo un mayor autoconcepto físico en los hombres que en las mujeres. Las dimensiones académicas y familiar no tienen diferencias según el género.

En cuanto al componente narrativo - cualitativo del grupo focal se obtuvo se identifica con mayor prevalencia los siguientes códigos que describen lo siguiente:

Se identifica que el desempeño académico está establecido mediante el rendimiento por género. Para la dimensión social es considerado su intensidad por las capacidades relaciones sociales por el nivel de amistad que tienen tanto hombres y mujeres, donde se identifica que las mujeres son más meticulosas al hacer amistad dando la diferencia de habilidades sociales entre ambos géneros, y la expresión pública de las emocionales que 
los estereotipos de género impuesto por la sociedad. Relacionando a la dimensión de la emoción, la diferencia radica en la regulación emocional y la sensibilidad emocional, estos dos factores se relacionan con la educación en el hogar y la enseñanza del manejo emocional. Dentro de la dimensión física la diferencia se encuentra en el cuidado del aspecto personal entre géneros.

\section{CONCLUSIÓN O CONSIDERACIONES FINALES}

El objetivo del presente estudio fue analizar el autoconcepto en relación al género en una muestra de adolescentes de entre 16-17 años de edad, por lo que se pudo evidenciar que existe una diferencia representativa de las dimensiones del autoconcepto tanto en hombres como en mujeres, se identificó que las mujeres tienen un autoconcepto mayor en la dimensión social y emocional y los hombres tienen un autoconcepto mayor en las dimensiones físico, finalmente la dimensión de autoconcepto académico y familiar es bueno en los dos géneros. Encontrando investigaciones previas que arrojan resultados similares, Núñez \& Albo (2004) indicaron que existe diferencias de género en al autoconcepto, a nivel global, los hombres presentan un mejor autoconcepto que las mujeres exceptuando la dimensión académica, Esnaola (2006) demuestra que los chicos obtienen estadísticamente mejores puntuaciones en el autoconcepto general, emocional, y físico general, Ingles et al., (2009) hallaron que los chicos puntuaron significativamente más alto en las dimensiones de habilidades física, mientras que las chicas presentaron puntuaciones más altas en relaciones con los padres, Padilla et al., (2010) presentaron que los chicos destacan en el autoconcepto emocional, físico, social y global, mientras que las chicas lo hacen en los planos familiar y académico, Vicent et al., (2015) evidenciaron diferencias de género para las dimensiones de autoconcepto, en los chicos en el autoconcepto físicos, estabilidad emocional y relaciones con los padres. Zubeldia et al., (2018) demuestran una media significativamente mayor en las mujeres en relación al autoconcepto social y académico, mientras que en los hombres presenta mayor puntaje en el autoconcepto físico y Roa et al., (2020) como resultado muestran persistencia de que las mujeres presentan un autoconcepto menor en comparación a los hombres.

Siendo así que se evidencia en general una diferencia significativa del autoconcepto

global, hay dimensiones que predomina más en los hombres como es el físico y en las mujeres como es el emocional, sin embargo, hay diferencias de los resultados de la investigación con las anteriores pudiendo ser por la población donde fueron desarrollados 
los proyectos, además de los años de aplicación, ya que los resultados cronológicamente desde el año 2010 hasta la actualidad pueden haber sido influidos por la visión de la sociedad que tiene hacia el género. Y en la dimensión académica y familiar que existe una equidad en hombres y mujeres pudiendo ser por el cambio de estilo de enseñanza aprendizaje siendo la modalidad virtual, haciendo que tanto hombres como mujeres tengan el mismo tipo de educación fuera de la influencia social, quedarse en casa incrementando la relación familiar.

Estos resultados confirman la hipótesis del estudio, tal y como se esperaba, hay diferencia del autoconcepto en hombres y mujeres, pero se ven diferenciados los resultados de este estudio con los previos posiblemente por la población donde se ha aplicado las investigaciones, de esta forma, los resultados del presente estudio apuntan a un mantenimiento de los patrones estereotipados de género, y que fenómenos representativos sean sociales o naturales como el caso de una Pandemia pueden influir en la visión que tienen los sujetos de sí mismos.

Sería interesante para futuras investigaciones profundizar en el análisis de la sociedad y estereotipos, ya que la diferencia de las dimensiones y su predominancia en hombres y mujeres puede estar influido por el contexto, logrando identificar si ha venido evolucionando la sociedad hacia la igualdad de género y la manera como se visualiza el adolescente.

\section{LISTA DE REFERENCIAS}

Aroca, C., Bellver, C., \& Alba. (2006). La teoría del aprendizaje social como modelo explicativo de la violencia filio-parental.

Esnaola, I. (2006). Diferencias de sexo en el autoconcepto de los adolescentes. INFAD Revista de Psicología, 2(1), 67-75.

Etchezahar, E. (2014) La construcción social del género desde la perspectiva de la Teoría de la Identidad Social. Ciencia, Docencia y Tecnología, 25(49) https://www.redalyc.org/pdf/145/14532635005.pdf

García, F., Musitu, G. (2021). Manual AF-5 Autoconcepto forma 5. TEA

Gonzalez (1990). Los estereotipos como factor de socialización en el género. Comunicar,12 https://www.redalyc.org/pdf/158/15801212.pdf

González, M., \& Taurón, J. (1992). Autoconcepto y rendimiento académico.

Hernández, R. Fernández, C. y Baptista, P. (2014). Metodología de la investigación 
Ingles, C., Pastor, Y., Torregrosa, M., Redondo, J., \& Garcia, J. (2009). Diferencias en funcion del género y el curso académico en dimensiones del autoconcepto: Estudio con una muestra de adolescentes españoles. Anuario de Psicologia, 40(2), 271-288.

Loperena, M. (2008) El autoconcepto en niños de cuatro a seis años. Tiempo de Educar, $9(18)$

https://www.redalyc.org/pdf/311/31111811006.pdf

Ramos, C. (2015). Los paradigmas de la investigación científica. 23(1). 9 - 17

Melero, N. (2010). Reivindicar la igualdad de mujeres y hombres en la sociedad: una aproximación al concepto de género. Barataria

Núñez, J., Albo, J. (2004). Psicología de la persona sin hogar: estilos de apego y autodeterminacion View project Contextual factors and human motivation View project. https://www.researchgate.net/publication/336533450

Padilla, T., Gómez, S., \& Suarez, M. (2010). Diferencias de género en el autoconcepto general y académico de estudiantes de 4o de ESO. Revista de Educacion, 352, 495-515. https://idus.us.es/xmlui/handle/11441/42557

Palladares, M. (2012). La cultura de genero en la actualidad: actitudes del colectivo adolescente hacia la actualidad. Tendencias pedagógicas

Roa, K., Castillo, H., Valdivia, M., Briseño, M., Gómez, M., Navarro, M., Annjeanette, M., Rivera, L., \& Conejeros, M. (2020). Autoconcepto y alta capacidad: influencia de estereotipos de género y programas de enriquecimiento.

Rocha, T. (2009) Desarrollo de la Identidad de Género desde una Perspectiva PsicoSocio-Cultural: Un Recorrido Conceptual. Interamerican Journal of Psychology, 43(2) https://www.redalyc.org/pdf/284/28412891006.pdf

Solís, A,. (2010). La perspectiva de género en la educación. https://www.rediech.org/inicio/images/k2/Desarrollo2-articulo2-5.pdf

Vargas, Z. (2009). La investigación aplicada: una forma de conocer las realidades con evidencia científica. Educacion, 33 (1), 155 -165

Vaz, A,. (2015). Lo que un buen autoconcepto puede hacer por nosotros. Medicina psicosomática y psiquiatría de enlace revista iberoamericana de psicosomática.

Vicent, M., Lagos, N., Gonzálvez, C., Inglés, C., García, J., \& Gomis, N. (2015). Diferencias de género y edad en autoconcepto en estudiantes adolescentes 
chilenos. Revista de Psicología, 24(1). https://doi.org/10.5354/07190581.2015 .36752

Zubeldia, M., Díaz, M., \& Goñi, E. (2018). Autoconcepto, atribuciones causales y ansiedad-rasgo del alumnado de conservatorio. Diferencias asociadas a la edad y al género. Psychology, Society and Education, 10(1), 79-102. https://doi.org/10.25115/psye.v10i1.1048 